ESAIM: PROCEEDINGS, October 2011, Vol. 32, p. 23-30

E. Cancès, N. Crouseilles, H. Guillard, B. Nkonga, and E. Sonnendrücker, Editors

\title{
HYBRID MODEL FOR THE COUPLING OF AN ASYMPTOTIC PRESERVING SCHEME WITH THE ASYMPTOTIC LIMIT MODEL: THE ONE DIMENSIONAL CASE.*
}

\author{
Pierre Degond $^{1}$, Fabrice Deluzet ${ }^{1}$, Dario Maldarella ${ }^{2}$, Jacek Narski ${ }^{1}$, \\ Claudia Negulescu ${ }^{3}$ and Martin Parisot ${ }^{4}$
}

\begin{abstract}
In this paper a strategy is investigated for the spatial coupling of an asymptotic preserving scheme with the asymptotic limit model, associated to a singularly perturbed, highly anisotropic, elliptic problem. This coupling strategy appears to be very advantageous as compared with the numerical discretization of the initial singular perturbation model or the purely asymptotic preserving scheme introduced in previous works [3,5]. The model problem addressed in this paper is well suited for the simulation of a plasma in the presence of a magnetic field, whose intensity may vary considerably within the simulation domain.
\end{abstract}

\section{INTRODUCTION}

This paper is devoted to the development of an efficient numerical method for strongly magnetized plasmas, such as the ones occurring in a tokamak. Due to the large magnetic field, the medium is highly anisotropic and numerous models require the resolution of an anisotropic elliptic equation (see $[2,6]$ ). In this framework, the anisotropy ratio is related to the reciprocal of the dimensionless cyclotron frequency. The model problem addressed in the present paper is for example well suited for the investigation of the Euler-Lorentz system in the drift limit (see $[2,6])$, the computation of the momentum component aligned with the magnetic field requiring the resolution of an anisotropic elliptic equation, in which the dominant part of the operator is supplemented with either Neumann or Periodic boundary conditions. The purpose is then to develop a numerical scheme to compute accurately and rapidly the solution of this anisotropic elliptic problem for considerably varying

\footnotetext{
* This work has been supported by the Marie Curie Actions of the European Commission under the contract DEASE (MEST-CT2005-021122), by the French 'Commissariat à l'Energie Atomique (CEA)' under contracts ELMAG (CEA-Cesta 4600156289), and GYRO-AP (Euratom-CEA V 3629.001), by the Agence Nationale de la Recherche (ANR) under contract IODISEE (ANR09-COSI-007-02), by the Fondation Sciences et Technologies pour l'Aéronautique et l'Espace (STAE) under contract PLASMAX (RTRA-STAE/2007/PF/002) and by the Scientific Council of the Université Paul Sabatier, under contract MOSITER. Support from the French magnetic fusion programme fédération de recherche sur la fusion par confinement magnétique is also acknowledged. The first two authors would like to express their gratitude to G. Gallice and C. Tessieras from CEA-Cesta for bringing their attention to this problem, to G. Falchetto, X. Garbet and M. Ottaviani from CEA-Cadarache, for their constant support to this research program.

${ }^{1}$ Université de Toulouse, UPS, INSA, UT1, UTM, Institut de Mathématiques de Toulouse, F-31062; Toulouse, France; CNRS, Institut de Mathématiques de Toulouse UMR 5219, F-31062 Toulouse, France.

2 Department of Mathematics and CMCS; University of Ferrara, Via Macchiavelli; 35 I-44100 Ferrara, Italy.

3 CMI/LATP (UMR 6632), Université de Provence; 39, rue Joliot Curie; 13453 Marseille Cedes, France.

${ }^{4}$ Project-Team SIMPAF-INRIA Lille-Nord Europe; Park Plazza, 40 avenue Halley; F-59650 Villeneuve d'Ascq cedex, France.
}

(C) EDP Sciences, SMAI 2011 
anisotropy values. Indeed, the anisotropy is assumed large in some sub-regions of the simulation domain, elsewhere of magnitude one. The final goal is to propose a coupling strategy between an Asymptotic-Preserving reformulation of the initial problem, used in the regions where the anisotropy is not too large, and the limit model for very large anisotropies. This Limit model is obtained from the AP-formulation by letting the anisotropic parameter, defined as the reciprocal of the anisotropy ratio, tend to zero.

The paper is then organized as follows. The singularly perturbed model problem is stated in section 1 for a simplified one dimensional configuration. An Asymptotic Preserving (AP) reformulation, introduced in a previous work (see $[3,5]$ ), is then detailed. This AP-method combines a discretization of the singular perturbation problem for intermediate values of the anisotropy and a discretization of the limit problem for vanishing anisotropic parameter, being thus accurate independently on the intensity of the anisotropy. Note that these Asymptotic Preserving techniques have been first introduced in [7]. The coupling strategy with the Limit model is then addressed in section 2 and detailed for both the singular perturbation problem and the AP-method. Finally the numerical schemes are briefly presented and first numerical experiments are discussed in section 3 .

The coupling strategy for the general multidimensional problem, as well as the detailed mathematical and numerical analysis, shall be the objective of the forthcoming work [4].

\section{The one Dimensional model problem And its Asymptotic Preserving FORMULATION.}

\subsection{The singular perturbation problem}

The aim of this paper is the study of the following one-dimensional model problem. Let $\Omega_{z}=[-1,1]$ be the computational domain, $z$ being the space variable. The singular perturbation problem (P-model) reads then

$$
(P)\left\{\begin{array}{l}
-\partial_{z}\left(\frac{1}{\varepsilon(z)} \partial_{z} u_{\varepsilon}\right)+u_{\varepsilon}=f, \quad z \in \Omega_{z}, \\
\frac{1}{\varepsilon(1)} \partial_{z} u_{\varepsilon}(1)=g^{+}, \quad \frac{1}{\varepsilon(-1)} \partial_{z} u_{\varepsilon}(-1)=g^{-},
\end{array}\right.
$$

where $u_{\varepsilon}: \Omega_{z} \rightarrow \mathbb{R}$ is the unknown of the problem and $\varepsilon$ is a given regular and positive function with large variations on the computational domain. In the rest of this document, the system (1) will be referred to as the Singular Perturbation model ( $P$-model), its weak formulation is precised by

Theorem 1. Let $f \in L^{2}\left(\Omega_{z}\right)$ and $\varepsilon \in L^{\infty}\left(\Omega_{z}\right)$ such that $0<\varepsilon(z)<1$ for almost all $z \in \Omega_{z}$. Then there exist a unique solution $u_{\varepsilon} \in H^{1}\left(\Omega_{z}\right)$ of the P-model, verifying the weak form

$$
\text { (P) } \int_{\Omega_{z}} \varepsilon^{-1} \partial_{z} u_{\varepsilon} \partial_{z} \psi d z+\int_{\Omega_{z}} u_{\varepsilon} \psi d z=\int_{\Omega_{z}} f \psi d z+g^{+} \psi(1)-g^{-} \psi(-1), \quad \forall \psi \in H^{1}\left(\Omega_{z}\right) .
$$

Note that a straight discretization of the system (2) for $0<\varepsilon \ll 1$ produces a linear system with a large conditioning number. Indeed, assuming formally $\varepsilon=0$ in the whole domain, system (1) leads to

$$
\left\{\begin{array}{l}
\partial_{z z}^{2} u=0, \quad \text { in } \Omega_{z}, \\
\partial_{z} u=0, \quad \text { in } \partial \Omega_{z} .
\end{array}\right.
$$

This system has an infinite amount of solutions since it is verified by all the constants. For this reason the discretized system (1) is expected to have a conditioning number that blows up with vanishing values of $\varepsilon$. To circumvent this difficulty an asymptotic preserving scheme has been introduced in $[3,5]$. The guidelines for the construction of this AP-scheme are briefly sketched here, and we refer to the above articles for the full details. 


\subsection{Asymptotic preserving reformulation of the problem}

The AP-scheme is obtained by reformulating the system (1) in such a manner, that the limit $\varepsilon \rightarrow 0$ becomes regular. It relies on a decomposition of the solution into its mean part corrected by a fluctuation as defined by

$$
u_{\varepsilon}(z)=\bar{u}_{\varepsilon}+u_{\varepsilon}^{\prime}(z), \quad \bar{u}_{\varepsilon}=\frac{1}{L_{z}} \int_{\Omega_{z}} u_{\varepsilon}(z) d z, \quad \text { where } L_{z}=\operatorname{mes}\left(\Omega_{z}\right) .
$$

The fluctuating part of the solution has a zero mean value, a fundamental property for the AP-scheme. Integrating now (1) along the anisotropy direction gives an equation for the mean part, for all $\varepsilon$-values:

$$
\bar{u}_{\varepsilon}=\bar{f}+\frac{g^{+}-g^{-}}{L_{z}} .
$$

The equation verified by the fluctuating component can be obtained by taking the difference of equations (1) and (4), leading to

$$
\left\{\begin{array}{l}
-\partial_{z}\left(\varepsilon^{-1} \partial_{z} u_{\varepsilon}^{\prime}\right)+u_{\varepsilon}^{\prime}=f^{\prime}-\frac{g^{+}-g^{-}}{L_{z}}, \quad \text { in } \Omega_{z}, \\
\varepsilon^{-1}(1) \partial_{z} u_{\varepsilon}^{\prime}(1)=g^{+}, \quad \varepsilon^{-1}(-1) \partial_{z} u_{\varepsilon}^{\prime}(-1)=g^{-}, \\
\int_{\Omega_{z}} u_{\varepsilon}^{\prime} d z=0 .
\end{array}\right.
$$

This system has a unique solution for all values of $\varepsilon$. The AP-method is composed of equations (4) and (5), providing respectively the mean and the fluctuating parts of the solution. A Lagrangian multiplier is introduced in the weak formulation of the AP-formulation, in order to cope with the constraint $\bar{u}_{\varepsilon}^{\prime}=0$

Theorem 2. There exist a unique solution $\left(u_{\varepsilon}^{\prime}, \bar{u}_{\varepsilon}, \lambda\right) \in H^{1}\left(\Omega_{z}\right) \times \mathbb{R} \times \mathbb{R}$ of the Asymptotic Preserving reformulation (AP-model)

$$
\begin{gathered}
\bar{u}_{\varepsilon}=\frac{1}{L_{z}} \int_{\Omega_{z}} f d z+\frac{g^{+}-g^{-}}{L_{z}}, \\
\left\{\begin{array}{l}
\int_{\Omega_{z}} \varepsilon^{-1} \partial_{z} u_{\varepsilon}^{\prime} \partial_{z} \psi d z+\int_{\Omega_{z}} u_{\varepsilon}^{\prime} \psi d z+\lambda \int_{\Omega_{z}} \psi d z= \\
\int_{\Omega_{z}}\left(f^{\prime}-\frac{g^{+}-g-}{L_{z}}\right) \psi d z+g^{+} \psi(1)-g^{-} \psi(-1), \quad \forall \psi \in H^{1}\left(\Omega_{z}\right) \\
\int_{\Omega_{z}} u_{\varepsilon}^{\prime} d z=0 .
\end{array}\right.
\end{gathered}
$$

Moreover, $u_{\varepsilon}:=\bar{u}_{\varepsilon}+u_{\varepsilon}^{\prime}$ is the unique solution of the (P)-problem (1) and $\lambda=0$.

The results of this section are obvious implications of the results obtained in $[3,5]$.

\section{The COUPling STRATEGy.}

Let us now assume that the anisotropy function is very small in one sub-domain and of magnitude one in the rest of the domain. The aim shall be to couple two different models, associated with the two sub-regions, the coupling strategy being based on Dirichlet-to-Neumann interface conditions. Let for this $\Omega_{z}=\Omega_{z}^{+} \cup \Omega_{z}^{-}$with $\Omega_{z}^{-}=[-1,0]$ and $\Omega_{z}^{+}=[0,1], L_{z}^{-}=\operatorname{mes}\left(\Omega_{z}^{-}\right), L_{z}^{+}=\operatorname{mes}\left(\Omega_{z}^{+}\right)$, and let the function $\varepsilon$ verify $\varepsilon(z)=\delta \chi(z), \forall z \in$ $\Omega_{z}^{-}$with $0<\delta \ll 1$ and $0<\chi(z)<1, \forall z \in \Omega_{z}^{-}$. For simplicity reasons, we shall omit here the $\varepsilon$ index of the solution $u_{\varepsilon}$ of the $(\mathrm{P})$-model, but shall denote it by $u^{+}$in $\Omega_{z}^{+}$resp. $u^{-}$in $\Omega_{z}^{-}$.

The P-problem can thus be rewritten in $\Omega_{z}^{-}$as

$$
\left\{\begin{array}{c}
-\partial_{z}\left(\chi^{-1} \partial_{z} u^{-}\right)+\delta u^{-}=\delta f, \quad z \in \Omega_{z}^{-} \\
\chi^{-1}(-1) \partial_{z} u^{-}(-1)=\delta g^{-}
\end{array}\right.
$$


The limit regime in the sub-domain $\Omega_{z}^{-}$can be investigated by letting $\delta \rightarrow 0$, which yields $\partial_{z} u^{-}=0$ in $\Omega_{z}^{-}$. The solution $u^{-}$in this limit regime is constant and totally determined by its value at the interface $z=0$,

$$
u^{-}(z)=u^{-}(0), \quad \forall z \in \Omega_{z}^{-} .
$$

This is the big advantage of the coupling strategy: the solution in the small $\varepsilon$-region does not depend on the coordinate aligned with the anisotropy. Its computation is then provided by a simplified system of equations as compared to the original problem, leading thus to considerable gain in simulation time.

A boundary condition at the sub-domain interface has to be provided for the computation of the solution in $\Omega_{z}^{+}$, ensuring by this mean the coupling of the two models. In this aim the equation (1) is integrated on $\Omega_{z}^{-}$ giving, thanks to property (7)

$$
L_{z}^{-} u^{-}(0)-\varepsilon^{-1}(0) \partial_{z} u^{-}(0)=\int_{\Omega_{z}^{-}} f(z) d z-g^{-} .
$$

Due to continuity arguments, the computation of the solution in $\Omega_{z}^{+}$is then approximated by the following problem

$$
(P-L)\left\{\begin{array}{l}
-\partial_{z}\left(\varepsilon^{-1} \partial_{z} u^{+}\right)+u^{+}=f, \quad \text { in } \Omega_{z}^{+}, \\
\varepsilon^{-1}(1) \partial_{z} u^{+}(1)=g^{+}, \\
-\varepsilon^{-1}(0) \partial_{z} u^{+}(0)+L_{z}^{-} u^{+}(0)=\int_{\Omega_{z}^{-}} f(z) d z-g^{-},
\end{array}\right.
$$

with $u^{-}(z)=u^{+}(0)$ in $\Omega_{z}^{-}$. The weak formulation of the coupled P-L model (P-model coupled to the Limit model) is given by: Find $\left(u^{+}, u^{-}\right) \in H^{1}\left(\Omega_{z}^{+}\right) \times H^{1}\left(\Omega_{z}^{-}\right)$, such that

$$
\begin{gathered}
\int_{\Omega_{z}^{+}} \varepsilon^{-1} \partial_{z} u^{+} \partial_{z} \phi d z+\int_{\Omega_{z}^{+}} u^{+} \phi d z=\int_{\Omega_{z}^{+}} f \phi d z+g^{+} \phi(1) \\
-L_{z}^{-} u^{+}(0) \phi(0)+\left(\int_{\Omega_{z}^{-}} f d z-g^{-}\right) \phi(0), \quad \forall \phi \in H^{1}\left(\Omega_{z}^{+}\right), \\
u^{-}(z)=u^{+}(0), \quad \forall z \in \Omega_{z}^{-} .
\end{gathered}
$$

Comparable computations allow the derivation of the coupling strategy for the AP-formulation with the Limit-model. First, as a consequence of the property (7), we have $u^{-^{\prime}}(z)=u^{+^{\prime}}(0), \forall z \in \Omega_{z}^{-}$. The zero mean property of the fluctuation can then be transformed into :

$$
\int_{\Omega_{z}^{+}} u^{+^{\prime}}(z) d z=-L_{z}^{-} u^{+^{\prime}}(0)
$$

Finally the AP-formulation coupled to the limit system reads :

$$
(A P-L) \begin{cases}-\partial_{z}\left(\varepsilon^{-1} \partial_{z} u^{+^{\prime}}\right)+u^{+^{\prime}}=f^{\prime}-\frac{g^{+}-g^{-}}{L_{z}}, & \text { in } \Omega_{z}^{+}, \\ \varepsilon^{-1}(z) \partial_{z} u^{+^{\prime}}(z)=g^{+}, & \text {on } z=1, \\ L_{z}^{-} u^{+^{\prime}}(z)-\varepsilon^{-1}(z) \partial_{z} u^{+^{\prime}}(z)=\int_{\Omega_{z}^{-}} f^{\prime} d z-\frac{L_{z}^{-}}{L_{z}}\left(g^{+}-g^{-}\right)-g^{-}, & \text {on } z=0 \\ \int_{\Omega_{z}^{+}} u^{+^{\prime}}(z) d z=-L_{z}^{-} u^{+^{\prime}}(0) . & \end{cases}
$$


where $u^{-^{\prime}}(z)=u^{+^{\prime}}(0) \forall z \in \Omega_{z}^{-}$and $\bar{u}$ is computed thanks to equation (4). The weak formulation of this system is given by: Find $\left(u^{+^{\prime}}, u^{-^{\prime}}, \bar{u}, \lambda\right) \in H^{1}\left(\Omega_{z}^{+}\right) \times H^{1}\left(\Omega_{z}^{-}\right) \times \mathbb{R} \times \mathbb{R}$ solution of the AP-L model (AP formulation coupled to the Limit model)

$$
\begin{gathered}
\bar{u}=\frac{1}{L_{z}} \int_{\Omega_{z}} f d z+\frac{g^{+}-g^{-}}{L_{z}}, \\
\int_{\Omega_{z}^{+}} \varepsilon^{-1} \partial_{z} u^{+^{\prime}} \partial_{z} \phi d z+\int_{\Omega_{z}^{+}} u^{+^{\prime}} \phi d z+\lambda \int_{\Omega_{z}^{+}} \phi d z=\int_{\Omega_{z}^{+}} f^{\prime} \phi d z-\frac{g^{+}-g^{-}}{L_{z}} \int_{\Omega_{z}^{+}} \phi d z \\
+g^{+} \phi(1)+\left(\int_{\Omega_{z}^{-}} f^{\prime} d z-\frac{L_{z}^{-}}{L_{z}}\left(g^{+}-g^{-}\right)-g^{-}-L_{z}^{-} u^{+^{\prime}}(0)\right) \phi(0), \quad \forall \phi \in H^{1}\left(\Omega_{z}^{+}\right), \\
\int_{\Omega_{z}^{+}} u^{+^{\prime}} d z=-L_{z}^{-} u^{+^{\prime}}(0), \quad \forall z \in \Omega_{z}^{-} .
\end{gathered}
$$

The rigorous mathematical study of this coupling procedure shall be studied in [4].

\section{NumericAl Methods AND EXPERIMENTS}

A discretization based on the finite element method is briefly sketched, before presenting the results of some numerical computations.

The domain $\Omega_{z}$ is descretized via a cartesian mesh defined by $\left(z_{i}\right)_{\mid i \in \llbracket-N, N \rrbracket}$, where $N$ is the number of unknowns in half of the computational domain. The weak formulations associated to the P-model (2) and its AP-formulation (6) as well as the coupled P-L model (9) and the coupled AP-L formulation (10) are discretized using the classical $\mathbb{P}_{1}$ finite element approximation. Two types of quadrature formulae are used for the integral approximations: Newton-Côtes with 1 to 5 points and Legendre-Gauss with 2 to 5 points. The linear systems obtained after discretization are solved thanks to the same direct method (MUMPS [1]).

To study the numerical results obtained via the presented four methods, an analytical solution is constructed, defined by

$$
u(z)=10+\varepsilon(z) \cos (\pi z), \quad z \in \Omega_{z} .
$$

This function verifies the boundary conditions. The anisotropy function $\varepsilon$ is given by

$$
\varepsilon(z)=\varepsilon_{0} \exp (p(z-1)), \quad \text { in } \Omega_{z},
$$

where $p$ and $\varepsilon_{0}$ are two real parameters, the former being a positive value providing the variations of $\varepsilon$, the latter representing the maximal value of $\varepsilon$ in the domain. This expression of the function $\varepsilon$ allows to address large variations of the anisotropy.

The first simulation is run for a uniform anisotropy $\varepsilon(z)=\varepsilon_{0}(p=0)$. The $L_{2}$-error, computed between the exact analytic solution (11) and the four numerical solutions is displayed on figure 1(a), as a function of $\varepsilon_{0}$. The discretized P-model is observed to produce accurate approximations for large and intermediate $\varepsilon_{0}$ values, but the error rapidly increases for values smaller than $10^{-5}$. This feature has already been mentioned in precedent works $[3,5]$. It can be explained by the conditioning of the discretized P-model which blows up with vanishing $\varepsilon_{0}$. The AP-formulation produces comparable accurate approximations as the P-model, on the range of large $\varepsilon_{0}$-values. However, the accuracy of the AP-scheme is preserved on the whole range of $\varepsilon_{0}$ values, and decreases even linearly until a plateau is reached for $\varepsilon_{0} \sim 10^{-10}$. Below this value the error remains constant for smaller anisotropies. The approximation error for the AP scheme can be decomposed in two parts: the error approximation for the mean and for the fluctuating part of the solution. The mean part computation accuracy is only explained by the quadrature formula used to approximate the mean part of $f$. This error is related to $h_{z}^{m}$ and will be denoted as $\mathcal{O}\left(h_{z}^{m}\right)$, where $h_{z}$ is the mesh interval and $m$ is a positive integer. For the fluctuating part, a differential equation is solved with a $\mathbb{P}_{1}$ finite element scheme, providing an approximation precise up to $\mathcal{O}\left(h_{z}^{2}\right)$ if the error is measured with the $L_{2}$-norm. More precisely, the fluctuating part of the 


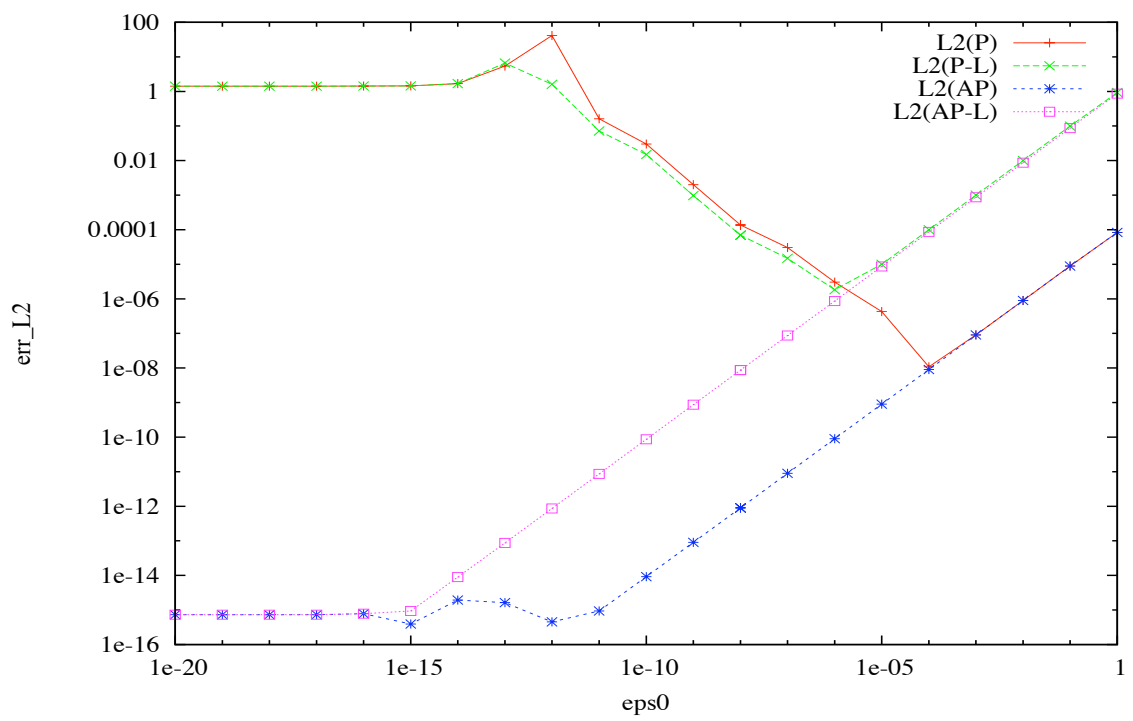

(a) Error in $L_{2}$-norm, as a function of $\varepsilon_{0}$ for $p=0$ (in log-scales).

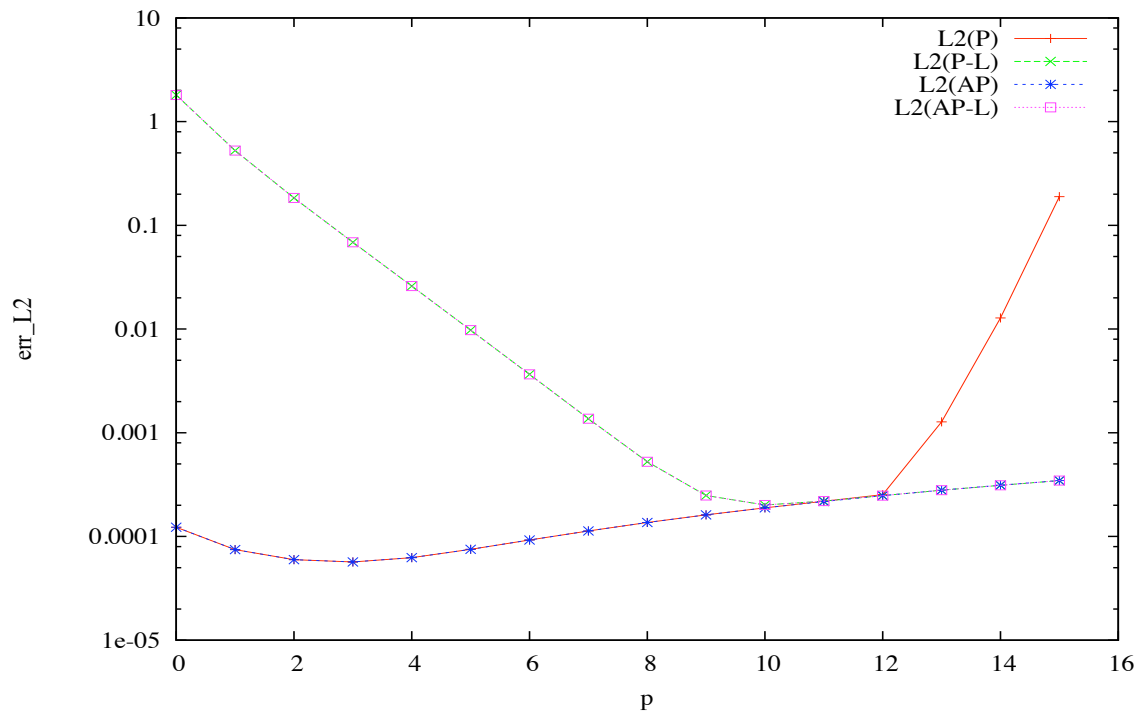

(b) Error in $L_{2}$-norm, as a function of $p$ for $\varepsilon_{0}=1$ (in log-scales).

FiguRE 1. Relative error $L_{2}$-norm for an anisotropy $\varepsilon(z)=\varepsilon_{0} \exp (p(z-1))$ between the exact solution and the numerical approximations. Four approaches are compared $(P)$, resp. $(A P)$ : Perturbation model, resp. Asymptotic Preserving formulation, on the whole domain, $(P-L)$ resp. $(A P-L)$ : the singular Perturbation model, resp. the Asymptotic Preserving formulation, on the right half space coupled with the limit model on the left half space $(A P-L)$. The computations are carried out on a mesh with 200 cells.

solution scaling as $\varepsilon_{0}$, the approximation error of the numerical scheme is comparable to $\varepsilon_{0} \mathcal{O}\left(h_{z}^{2}\right)$. For the AP-scheme the global error can then be defined as $\varepsilon_{0} \mathcal{O}\left(h_{z}^{2}\right)+\mathcal{O}\left(h_{z}^{m}\right)$. For the numerical experiments carried out here, the quadrature formulae provide an estimation of the integral terms with a precision much higher than the second order $(m>2)$. This explains the linear AP-scheme total error decrease with vanishing $\varepsilon_{0}$, 
for large and intermediate $\varepsilon_{0}$ values. In this range of $\varepsilon_{0}$-values, the global error is totally dominated by the fluctuating part approximation error $\left(\varepsilon_{0} \mathcal{O}\left(h_{z}^{2}\right)\right)$. For the smallest $\varepsilon_{0}$-values, the global error is dominated by the error approximation of the mean part and remains constant independently of the $\varepsilon_{0}$-values, explaining the plateau observed on figure 1 (a) for $\varepsilon_{0}<10^{-10}$. The analysis is quite the same for the AP-L scheme, but in this case an error due to the model has also to be taken into account. Indeed in one sub-domain, the fluctuating part of the solution is dropped, which translates into an error proportional to $\varepsilon_{0}$. The total error for the AP-L scheme is thus given by $\mathcal{O}\left(h_{z}^{m}\right)+\varepsilon_{0} \mathcal{O}\left(h_{z}^{2}\right)+\mathcal{O}\left(\varepsilon_{0}\right)$. The mesh interval $h_{z}$ being small compared to 1 , the total error is totally dominated by $\varepsilon_{0}$ for the largest values of the anisotropy range. A linear decrease of the error, with vanishing $\varepsilon_{0}$, is thus also observed, until the contribution of the term $\mathcal{O}\left(h_{z}^{m}\right)$ becomes significant. The efficiency of the coupling strategy is demonstrated by these first experiments. Indeed, for small values of $\varepsilon_{0}$ the AP-scheme coupled with the limit model produces very accurate results for the solution. Not surprisingly, for intermediate and large values of $\varepsilon_{0}$ the precision of this approximation is poor compared with that of the AP-formulation used on the whole simulation domain, since it is computed with an approximated limit model on half of the computational domain. Note that the advantage of the coupling strategy consists of a significant computational time gain, since, in the limit regime, the solution has one degree of freedom less. However, this coupling strategy is expected to be efficient in configurations where the anisotropy ratio is large in one part of the simulation domain, while being small in the other.

Finally a second simulation is carried out with a non uniform anisotropy. The error as a function of the parameter $p$ is displayed on figure $1(\mathrm{~b})$. These simulations are performed with $\varepsilon_{0}=1$. The approximations computed thanks to either the (P-L) or the (AP-L) formulations are accurate for the largest $p$-values . For small values of this parameter, the anisotropy variations are not large enough to obtain small $\varepsilon$ in the sub-domain where the limit problem is used. This explains the poor quality of the approximations for vanishing values of $p$. The singular perturbation problem is observed to suffer from a significant lack of accuracy for large variations of the anisotropy. Note that the coupling strategy allows to fix this issue, since the (P-L) approximation is accurate for the largest value of the parameter. Finally the AP-scheme furnishes accurate computations on the whole range of $p$ values, its accuracy being comparable to the singular perturbation model for moderate anisotropies while being comparable to the hybrid model on the other part of the $p$-values range. All this should be investigated further in the forthcoming work [4].

\section{Conclusion And Perspectives}

The results presented in this work concern a coupling strategy between an Asymptotic-Preserving reformulation of a Singular Perturbation problem, with the associated Asymptotic Limit model. The coupling is performed via Dirichlet-to-Neumann interface conditions. The aim is to accelerate the resolution of a highly anisotropic elliptic equation, as compared to the entirely AP-resolution introduced in previous works. The first numerical results are presented in a simplified $1 \mathrm{D}$ configuration. The detailed study (mathematical and numerical) of a general 3D framework will be the objective of the future work [4].

\section{REFERENCES}

[1] P. Amestoy, I. Duff, and J.-Y. L'Excellent. Mumps multifrontal massively parallel solver version 2.0, 1998.

[2] S. Brull, P. Degond, and F. Deluzet. Degenerate anisotropic elliptic problems and magnetized plasma simulations. To be submitted.

[3] P. Degond, F. Deluzet, A. Lozinski, J. Narski, and C. Negulescu. Duality-based asymptotic-preserving method for highly anisotropic diffusion equations. arXiv:1008.3405v1, 2010.

[4] P. Degond, F. Deluzet, D. Maldarella, J. Narski, C. Negulescu, and M. Parisot. Hybrid model for the coupling of an asymptotic preserving scheme with the asymptotic limit model. in preparation.

[5] P. Degond, F. Deluzet, and C. Negulescu. An asymptotic preserving scheme for strongly anisotropic elliptic problems. Multiscale Model. Simul., 8(2):645-666, 2010.

[6] P. Degond, F. Deluzet, A. Sangam, and M.-H. Vignal. An asymptotic-preserving scheme for the Euler equations in a strong magnetic field. J. Comput. Phys., 228(10):3540-3558, 2009. 
[7] S. Jin. Efficient asymptotic-preserving (AP) schemes for some multiscale kinetic equations. SIAM J. Sci. Comput., 21(2):441454, 1999. 